\title{
A young man with progressive esophageal neoplasms
}

\author{
Yaxing Shen ${ }^{1}$, Jianfei Shen ${ }^{2}$, Kevin Phan $^{3,4}$, David Tian ${ }^{5}$, Thomas A. D’Amico ${ }^{6}$, Mark F. Berry ${ }^{7}$, \\ Shanda H. Blackmon ${ }^{8}$, Shari L. Meyerson ${ }^{9}$, Xavier Benoit D'Journo ${ }^{10}$, Yi-Jen Chen ${ }^{11}$, Garrett Baron ${ }^{12}$, \\ Yingyong Hou ${ }^{13}$, Lijie Tan ${ }^{1}$; written on behalf of AME Esophageal Cancer Collaborative Group
}

\begin{abstract}
${ }^{1}$ Department of Thoracic Surgery, Zhongshan Hospital of Fudan University, Shanghai 200032, China; ${ }^{2}$ Taizhou Hospital of Zhejiang Province, Wenzhou Medical University, Wenzhou 325000, China; ${ }^{3}$ Collaborative Research (CORE) Group, Sydney, Australia; ${ }^{4}$ Faculty of Medicine, University of Sydney, Sydney, Australia; ${ }^{5}$ Collaborative Research (CORE) Group, Macquarie University, Sydney, Australia; ${ }^{6}$ Department of Surgery, ${ }^{7}$ Division of Thoracic Surgery, Department of Surgery, Duke University Medical Center, Durham, North Carolina, USA; ${ }^{8}$ Thoracic Surgery, Department of Surgery, The Mayo Clinic, Rochester, MN 55905, USA; 'Division of Thoracic Surgery, Feinberg School of Medicine, Northwestern University, Chicago, IL 60611, USA; ${ }^{10}$ Department of Thoracic Surgery, North Hospital, Aix-Marseille University, Chemin des Bourrely, 13915 Marseille cedex 20 , France; ${ }^{11}$ Division of Radiation Oncology, City of Hope National Medical Center, Duarte, CA 91010, USA; ${ }^{12}$ School of Medicine, Wayne State University, Detroit, MI 48201, USA; ${ }^{13}$ Department of Pathology, Zhongshan Hospital, Fudan University, Shanghai 200032, China

Correspondence to: Lijie Tan, MD. Department of Thoracic Surgery, Zhongshan Hospital of Fudan University, 180 Fenglin Road, Shanghai 200032, China. Email: tan.lijie@zs-hospital.sh.cn.
\end{abstract}

Submitted Jul 24, 2017. Accepted for publication Oct 12, 2018.

doi: $10.21037 /$ jtd.2018.10.56

View this article at: http://dx.doi.org/10.21037/jtd.2018.10.56

Esophageal sarcomatoid carcinoma (ESC) is a rare malignancy, accounting for about $2 \%$ of esophageal malignancies. It is also referred to by numerous other names, including carcinosarcoma, pseudosarcoma, pseudosarcomatous squamous cell carcinoma, spindle cell carcinoma and polypoid carcinoma, suggesting that its pathological mechanism remains undefined. Its clinical and radiological manifestations are similar to other esophageal tumors. Barium esophagogram usually reveals the presence of a large polypoid mass in the lumen. While ESC is typically large, it has a relatively good prognosis, which may be explained by its exophytic growth rather than the infiltration into the esophageal wall. Surgical resection of the esophagus and stomach is the basis of early detection, effective treatment and long-term survival, and is the main treatment for ESC. However, as a rare disease, ESC is often misdiagnosed in clinical setting, especially in cases where a biopsy cannot provide sufficient specimens. In this article, we report a case of suspected ESC, in whom surgical treatment was avoided after international multidisciplinary consultations.

\section{Case presentation}

A 23-year-old man presented with "chest pain after meals" for 4 months and "acid reflux at night" for 2 months, which worsened one month ago. He visited a local hospital, where endoscopy revealed a $0.5-\mathrm{cm}$ ulceration $30 \mathrm{~cm}$ from the incisors, with "white moss" covering its surface. Symptomatic treatment was ineffective. The symptoms recurred frequently in the next 2 months, accompanied by blood in phlegm and acid reflux at night. Proton pump inhibitors (PPIs) were used for symptomatic treatment; however, the treatment was not effective and the symptoms worsened. The patient had no family history, or other past medical history. There were no other significant findings during physical examination. Chest CT suggested that the thoracic esophagus had thickened walls and a narrowed lumen, with clear boundaries with the adjacent organs and tissues (Figure 1).

From January 2014 to May 2014, the patient underwent multiple gastroscopic examinations and biopsy, which revealed that the lesion in the middle esophagus had rapidly increased from 0.5 to $4 \mathrm{~cm}$ (Figure $2 A, B, C, D$ ). At the end of January 2014, biopsy revealed squamous epithelial papillary hyperplasia and local ulcer formation and a pathological diagnosis of submucosal spindle cell hyperplasia was made.

In early April, another biopsy was performed, revealing the presence of inflammatory granulation, necrotic tissues and sporadic hyperplasia of fusiform cells in the mucosa, along with a small amount of abnormally shaped cells. The pathological diagnosis was fusiform cell tumor.

Three weeks later endoscopic ultrasound (EUS)-guided 

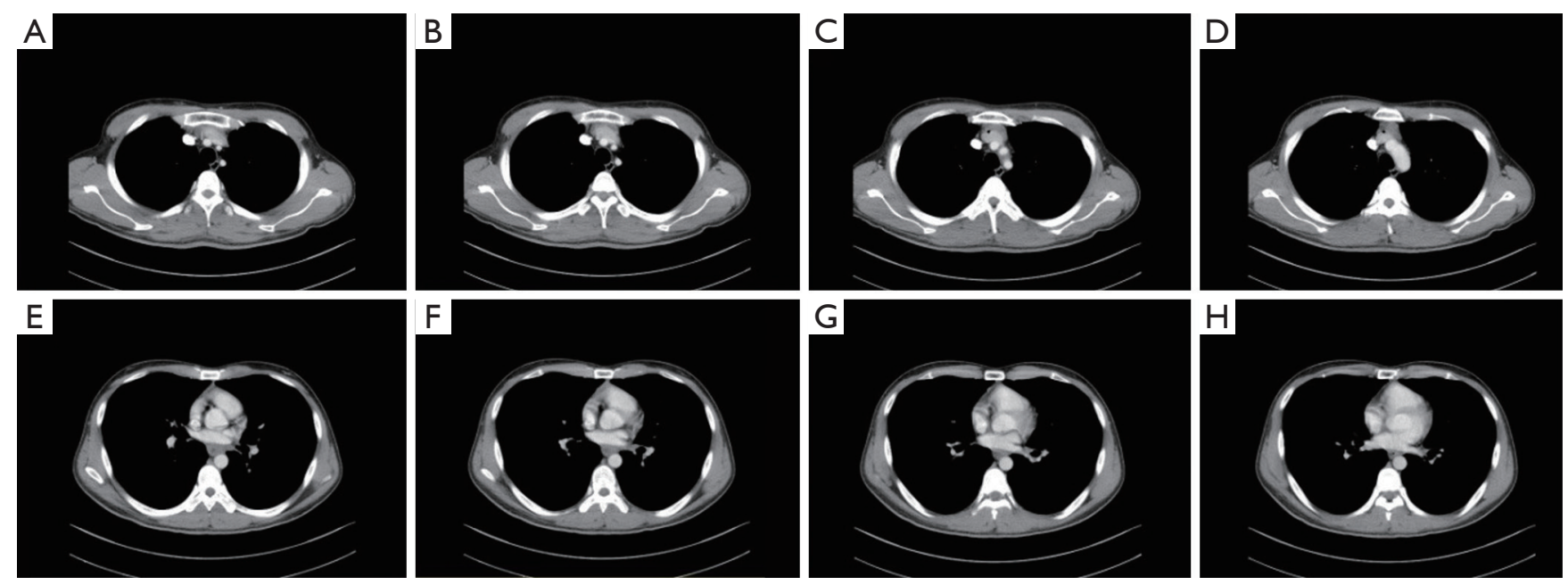

Figure 1 The thoracic esophagus has thickened walls and a narrowed lumen.
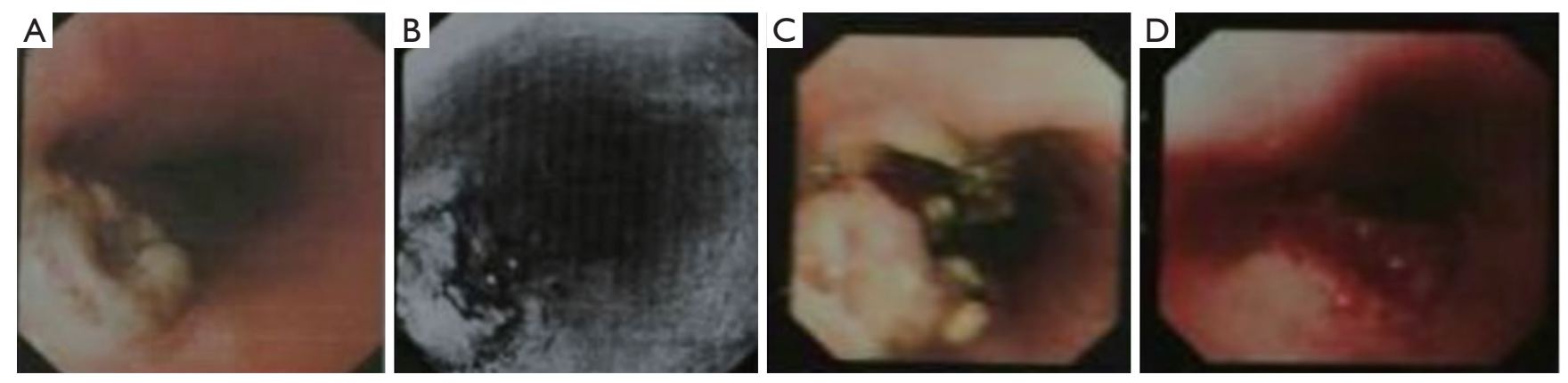

Figure 2 Endoscopic examination suggests a rapid increase of the lesion in the middle esophagus. (A) On January 30, 2014, gastroscopy revealed a $0.5-\mathrm{cm}$ pit $30 \mathrm{~cm}$ away from the incisors, with "white moss" covering its surface; (B) on April 4, 2014, gastroscopy revealed a 2.0-cm pit $30 \mathrm{~cm}$ away from the incisors, with the same white moss findings on the surface, but the lesion has now involved half of the esophagus; (C) on April 25, 2014, ultrasonic gastroscopy revealed a $3.0 \mathrm{~cm} \times 2.0 \mathrm{~cm}$ ulcer $29-32 \mathrm{~cm}$ away from the incisors, and the lesion had invaded the mucosal and submucosal layers; (D) on May 21, 2014, ultrasonic gastroscopy revealed a 4.0-cm longitudinal ulcer at the left front wall $27-32 \mathrm{~cm}$ away from the incisors, and the lesion had invaded all the esophageal wall layers.

biopsy revealed the presence of a spindle cell lesion: the cells were dense and moderately abnormal, with visible mitotic figures and infiltrative growth of the lesion into smooth muscle. The lesion was initially thought to be a malignant spindle cell tumor with some additional consideration as a pseudosarcomatous lesion.

In mid-May another biopsy revealed a spindle cell lesion: the cells were dense, with visible mitotic figures (3-5 mitoses per $10 \mathrm{HPF}$ ) (Figure 3). The lesion grew infiltratively and intermuscularly, showing a malignant tendency. Immunohistochemistry showed: CK7 $(80 \%++$ to +++$)$, CK $(70 \%+$ to ++$)$, desmin (tumor cell negative), Ki67 (30\% positive), a-SMA (tumor cell) (-), ALK-1 (-), and CK8
(-) (Figure 4), suggestive of the presence of a sarcomatoid carcinoma or fibrosarcoma.

\section{Expert opinions}

\section{Opinion 1}

If this is your patient and you are interested in my advice, I would do everything possible to not operate. The two settings that I have seen this in young patients is: (I) medication: ibuprofen taken at night without enough liquid; (II) embedded fish bone: is the CT scan sensitive enough to make sure there is not a foreign body embedded in the esophagus, such as a fish bone? I think it is worthwhile to 

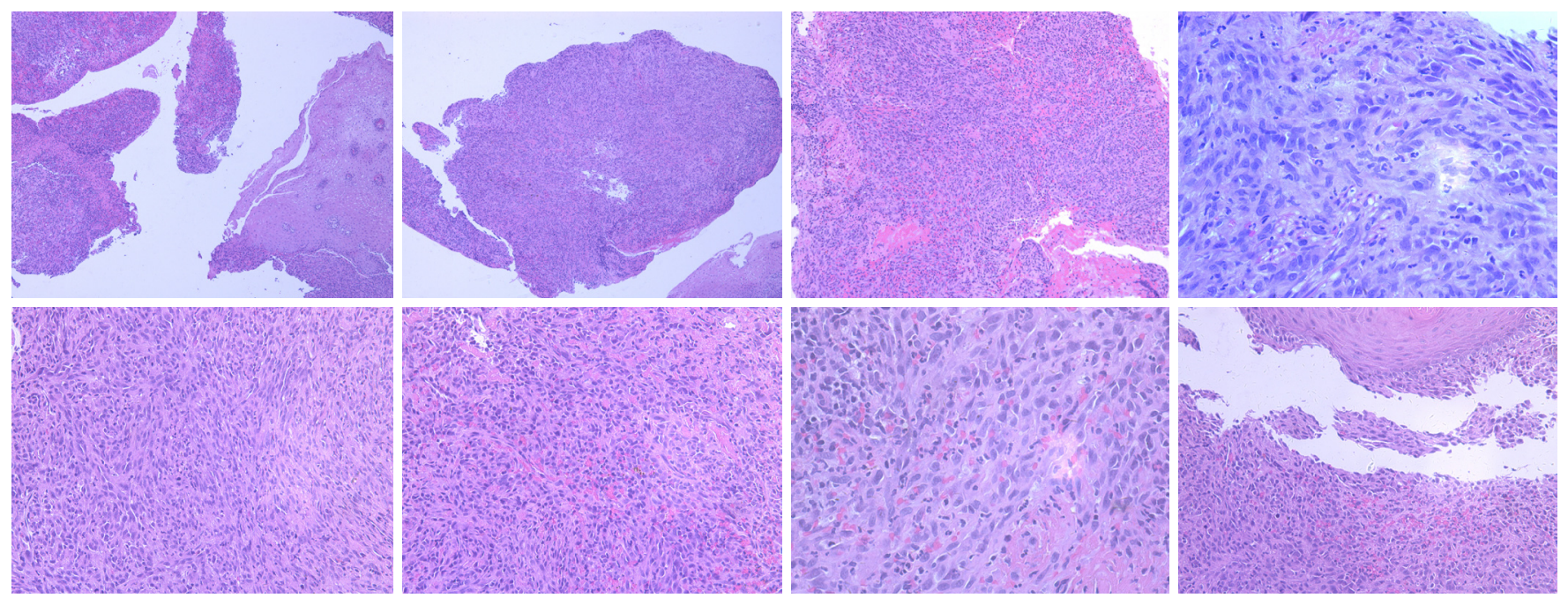

Figure $3 \mathrm{HE}$ staining revealed an esophageal spindle cell lesion: the cells were dense, with visible mitotic figures (3-5 mitoses per $10 \mathrm{HPF})$. The lesion grew infiltratively and intermuscularly, showing a malignant tendency. Magnification (from left to right, top to bottom): 50×, $50 \times, 100 \times, 400 \times, 200 \times, 200 \times, 400 \times, 200 \times$.

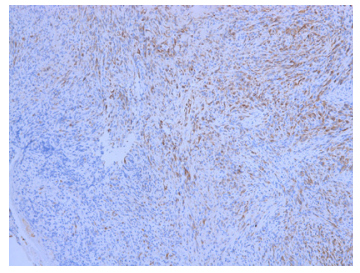

CK7

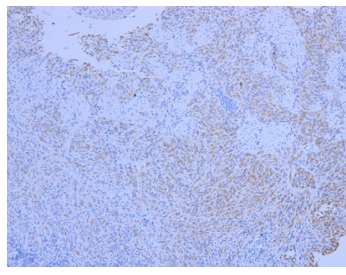

CK

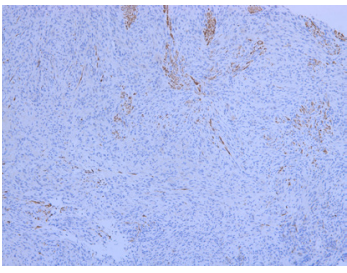

Desmin

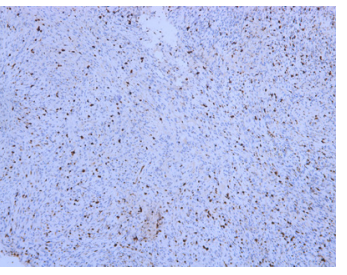

Ki67

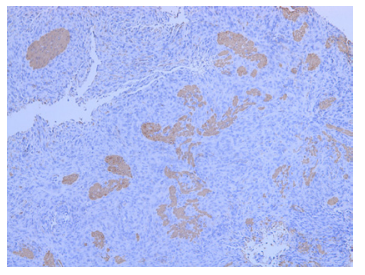

a-SMA

Figure 4 Immunohistochemistry showed: CK7 (80\%++ to ++), CK (70\% to ++), desmin (tumor cell negative), Ki67 (30\% positive), a-SMA (tumor cell negative). Magnification: $100 \times$.

repeat the endoscopy for two reasons: (I) look again for foreign body; it might be found with EUS; (II) follow for improvement vs deterioration.

\section{Opinion 2}

It does look like a tough case. Could this be an esophageal leiomyoma with overlying mucosal ulceration? It seems like resection is probably necessary, given the growth and persistent symptoms, even if the tumor/ulcer is benign. If it is a leiomyoma, I would try to enucleate the tumor rather than do an esophagectomy, though the ulceration is a little confusing. I'd consider getting a contrasted swallow exam (a barium swallow just to make sure that this is not some sort of chronic esophageal leak/abscess) and doing another endoscopy to get cultures of the ulcer, both fungal, AFB, and bacteria to make sure that there is not an infection that needs to be treated to get the ulcer to heal prior to attempting resection.

\section{Opinion 3}

I read this interesting and difficult case. First, I wonder if the patient has a dysphagia and weight loss. Second, because of the patient's young age, because of the tumor's rapid growth and if the patient remains symptomatic, I would hypothesize an esophageal aggressive fibromatosis (desmoid tumor). In this context, I would repeat the endoscopic biopsy and I would probably ask for a PET-CT. If I have some indications towards this diagnosis, I would perform an esophagectomy after discussion at the tumor board. This is a personal view. I hope this advice can help you. 


\section{Opinion 4}

I have reviewed the case, and although this is not a confirmed cancer case, I do believe the lesion is acting in a malignant fashion and would soon obstruct the esophagus. I have seen large benign (by histology)-appearing lesions within the esophagus that had malignant transformation or had invasive cancer within the papillary lesion but was not diagnosed until excision. I recommend esophagectomy with a cervical anastomosis. I am not sure if they have PET scan ability, but that may help to stage and if malignantappearing lymph nodes are identified, then they should be biopsied by EUS and if cancer is found within those nodes, then neoadjuvant therapy should be administered prior to resection. This was an interesting case.

\section{Opinion 5}

I have consulted with 2 of my colleagues, an expert gastrointestinal (GI) pathologist and an expert GI oncologist. Ulcerated masses in the mid-esophagus at this young age are unusual. Given the spindle cells appearance with entrapped epithelial elements, you may be looking at a gastrointestinal stromal tumor (GIST), leiomyoma, leiomyosarcoma, or an inflammatory pseudotumor (inflammatory myofibroblastic tumor). It is recommended to do CD117, DOG1 and ALK immunohistochemical staining (IHC) stains to confirm the diagnosis. In the meantime, given the rapid growth of the mass, surgery may be necessary before it starts invading into other mediastinal structures. I hope this would help you.

\section{Opinion 6}

Given the rapid progression, I would vote for surgery.

\section{Treatment}

According to the consultation opinions, we recommended the patient to undertake PET-CT, which revealed that the standard uptake value (SUV) was elevated at the 5th-7th thoracic vertebrae (SUVmax: 12.3) and the left hilar lymph node $(13.5 \mathrm{~mm} \times 11.3 \mathrm{~mm}$, SUVmax: 5.2). In addition, glucose metabolism increased in multiple lesions, accompanied by bone destruction (Figure 5).

IHC was performed again on the final specimen obtained by ultrasonic gastroscopic biopsy and the results were as follows: CK general $(60 \%+$ to ++), CAM5.2 (-), CK8 (-),
CD68 (+), SMA (-), MSA (-), DES (-), Ki67 (30\%+), ALK-1 $(-)$, CD34 (-), CK19 (partially+), Nestin (+), Vimentin $(++)$, CK7 (partially+), P63 (partially+), HCK (-), SDHA $(+)$, SDHB (+), LCK (partially+), and Keratin (-). The final pathological findings suggested the possibility of ESC.

In summation, according to the consultation opinions PET-CT was performed, which revealed esophageal tumor with multiple systemic metastasis while pathological findings suggested that the ESC was at an advanced stage. Thus, unnecessary resection of the esophagus was avoided in this young patient. However, we recommended the patient undergo biopsy of bone metastases, but the patient and his families refused to undertake a second biopsy and other treatments (e.g., radiochemotherapy). The patient is still alive after 3 years of follow-up.

\section{Conclusions}

In 1865, Virchow first described the concept of ESC, which accounted for $2 \%$ of esophageal cancers (1). It has a variety of names including carcinosarcoma, pseudosarcoma, pseudosarcomatous squamous cell carcinoma, spindle cell carcinoma, and polypoid carcinoma. The multitude of definitions and terms reflects that the pathogenic mechanism of ESC remains undefined other than its polyp-like characteristics. Histologically, ESC is a biphasic disorder: the epithelium-derived components are usually confined to several sites, and most parts of the tumor have a pleomorphic sarcoma-like appearance. The sarcoma components are often thought to be derived from tumor cells. However, some researchers believe there to be coexistence of two different malignant tumors, while others argued the sarcoma-like tissue could also be derived from epithelium $(2,3)$.

ESC is more likely to occur in males, particularly in populations aged $60-70$ years. About $60 \%$ of ESC are located in the middle esophagus, about $1 / 3$ at the distal end of the esophagus and less than $10 \%$ at the proximal end of esophagus (4). Patients with this malignancy often present with dysphagia, sometimes accompanied by chest pain and weight loss. These symptoms may last for several days to several months, but typically subside within 3 months. Since ESC is typically large, patients often develop obstructive symptoms in the early stage of the disease. Like many other esophageal tumors, ESC is usually diagnosed by radiologic and endoscopic examinations. Radiologically, it is often manifested as a huge mass but will not block the lumen. 

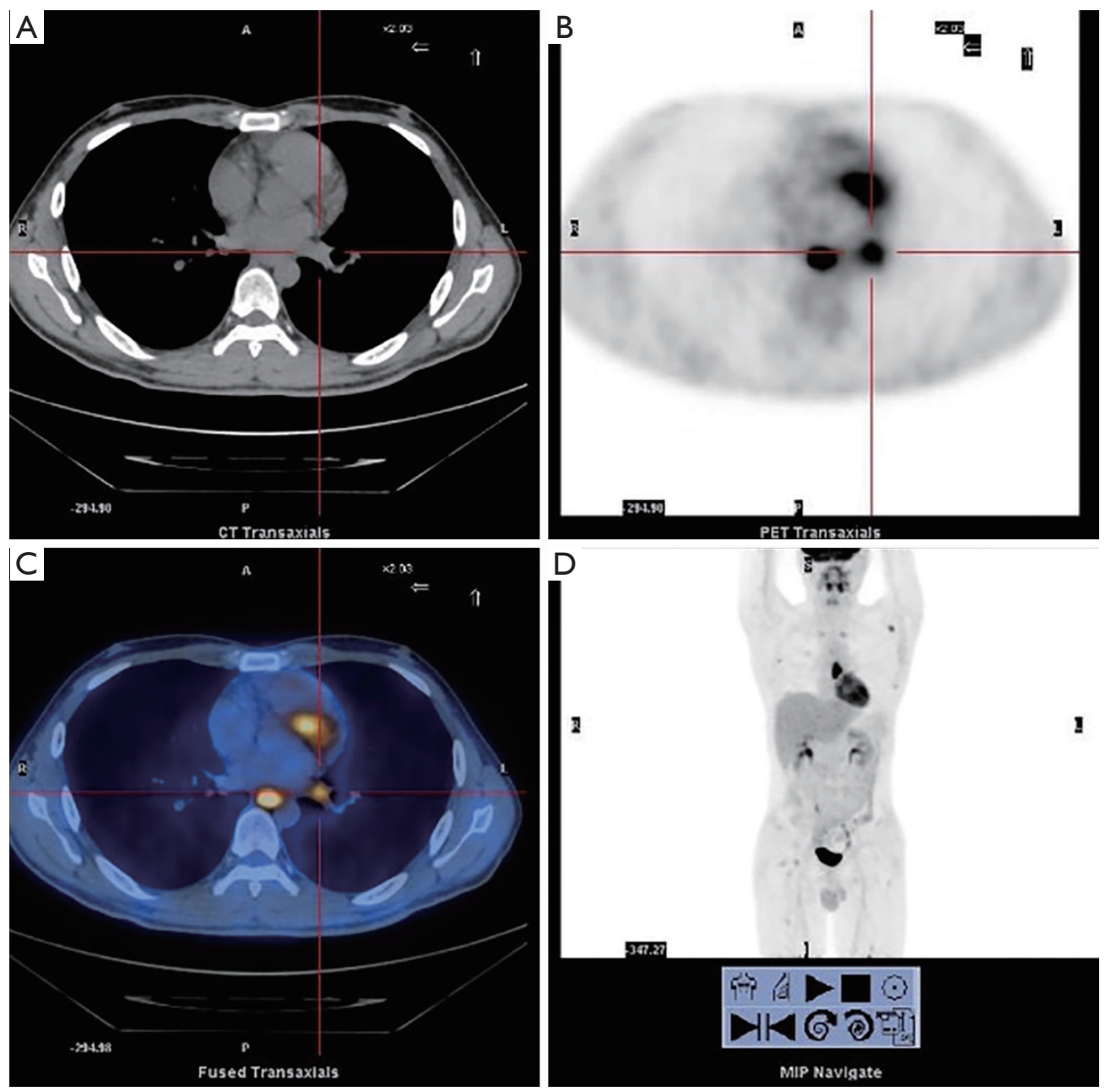

Figure 5 PET-CT revealed that the standard uptake value (SUV) was elevated at the 5th-7th thoracic vertebrae (SUVmax: 12.3 ) and the left hilar lymph node $(13.5 \mathrm{~mm} \times 11.3 \mathrm{~mm}$, SUVmax: 5.2). In addition, glucose metabolism increased in multiple lesions [bilateral clavicles, right ribs (2, 5, and 8), 10th-11th thoracic vertebrae, and bilateral femur], accompanied by bone destruction.

Under the microscope, these lesions show biphasic changes: it can be a mixture of cancer and malignant sarcoma-like substances, with the latter being the dominant component. The epithelium-derived tissue components are usually squamous epithelium, which coexist from in-situ or micro-infiltration to nested infiltration with spindle cells. Some researchers have also described its coexistence with adenocarcinoma or undifferentiated carcinoma (5). ESC can also have heterogeneous epithelial components such as squamous cell carcinoma and basal cell carcinoma. Ohtaka et al. found that ESC contained squamous and basal cell carcinoma, along with spindle cell proliferation (6). Amatya et al. also reported a case of heterogeneous canceration, in whom the basal and squamous cell carcinoma was the main component, followed by invasive and in-situ squamous cell carcinoma; notably, the sarcoma was characterized by striated muscle cell differentiation (7). It has also been reported that sarcoma is featured by the specific differentiation into real sarcomas such as malignant tissue cell tumors, leiomyosarcomas, chondrosarcomas, or osteosarcomas.

IHC shows that keratin is positive in the epithelial component of sarcomatoid carcinoma, while visinin is strongly positive in the interstitial components and occasionally may develop immune reactivity with keratin, although it is almost always manifested as focal and weakly positive during this period (7). The sarcomatoid cells may occasionally react with actin and desmin. Tumor cells in 
the transitional region between cancer and sarcoma tissues may have the same immune reactivity as the sarcoma components.

Wang et al. have studied the immunohistochemistry and pathogenesis of these tumors (8). Keratin was positive in nine cases and visinin was positive in five cases. In two cases, both keratin and visinin were weakly positive in the transitional region. The sarcoma components had positive visinin expression in ten cases and positive desmin expression in two cases. The authors conclude that tumor epithelial cells might be differentiated into spindle cells and non-epithelium-derived sarcoma (e.g., chondrosarcoma and leiomyosarcoma). Resection of the esophagus and stomach is the main treatment for ESC. The prognosis of ESC is better than that of common squamous cell carcinomas because of its exophytic growth pattern, rather than the infiltration into the esophageal wall. Although the tumor is relatively large, it is usually located at the intrinsic layer of the mucous membrane or above the submucosa. The depth of tumor invasion is an important risk factor for tumor metastasis and prognosis (4).

In summary, unnecessary esophagectomy was avoided in this young patient with esophageal tumor after an international multidisciplinary consultation. Unfortunately, however, the diagnosis of ESC has not yet been confirmed. A better understanding of ESC and active multidisciplinary consultations may increase the accuracy of medical decision-making.

\section{Acknowledgements}

We would like to express our thankfulness to Dr. Mari Mino-Kenudson for her pathology comments on the paper.

\section{Footnote}

Conflicts of Interest: The authors have no conflicts of interest to declare.

\section{References}

1. Odze R, Goldblum J. Variants of squamous cell carcinoma. In: Surgical Pathology of the GI Tract, Liver, Biliary Tract and Pancreas. 2nd editon. Philadelphia, PA: Saunders Elsevier, 2009:548-52.

2. Rosai J. Other types of esophageal carcinoma. In: Goldblum TR, Lamps LW, McKenney JK. Rosai and Ackerman's Surgical Pathology. 9th edition. St Louis, MO: CV Mosby Co, 2004:629-31.

3. Handra-Luca A, Terris B, Couvelard A, et al. Spindle cell squamous carcinoma of the oesophagus: an analysis of 17 cases, with new immunohistochemical evidence for a clonal origin. Histopathology 2001;39:125-32.

4. Klaus L, Henry A. Special variants of squamous cell carcinoma. In: Lewin KJ and Appelman HD. Tumors of the Esophagus and Stomach. Washington, DC: Armed Forces Institute of Pathology, 1996:83-91. Atlas of Tumor Pathology. 3rd series, fascicle 18.

5. Orsatti G, Corvalan AH, Sakurai H, et al. Polypoid adenosquamous carcinoma of the esophagus with prominent spindle cells: Report of a case with immunohistochemical and ultrastructural studies. Arch Pathol Lab Med 1993;117:544-7.

6. Ohtaka M, Kumasaka T, Nobukawa B, et al. Carcinosarcoma of the esophagus characterized by myoepithelial and ductal differentiations. Pathol Int 2002;52:657-63.

7. Amatya VJ, Takeshima Y, Kaneko M, et al. Esophageal carcinosarcoma with basaloid squamous carcinoma and rhabdomyosarcoma components with TP53 mutation. Pathol Int 2004;54:803-9.

8. Wang ZY, Itabashi M, Hirota T, et al. Immunohistochemical study of the histogenesis of esophageal carcinosarcoma. Jpn J Clin Oncol 1992;22:377-86.
Cite this article as: Shen Y, Shen J, Phan K, Tian D, D'Amico TA, Berry MF, Blackmon SH, Meyerson SL, D'Journo XB, Chen YJ, Baron G, Hou Y, Tan L; written on behalf of AME Esophageal Cancer Collaborative Group. A young man with progressive esophageal neoplasms. J Thorac Dis 2018;10(11):5985-5990. doi: 10.21037/jtd.2018.10.56 